\title{
Expanding access to contraceptive choice in Canada
}

\author{
Laura J McLeod, Helene Baldwin, Vanessa DeMelo \\ Faculty Reviewer: Shannon Arntfield, MD, FRCSC (Department of Obstetrics and Gynecology)
}

\section{ABSTRACT}

Every woman should possess the power to decide whether or not she chooses to become pregnant at any given point in her life. Unplanned pregnancy may have a huge impact on a woman's health, economic, and social well-being, and yet an estimated $41 \%$ of pregnancies worldwide are unintentional. There are a multitude of reversible contraceptive options available and many factors that contribute to a woman's decision regarding which method is right for her. Efficacy, effort and side effect profile are important considerations and vary widely between methods. Surprisingly, condoms and the oral contraceptive pill, which have relatively high typical use failure rates at $21 \%$ and $9 \%$ respectively and require frequent user action, are the most popular reversible contraceptive options in Canada. In contrast, intrauterine contraceptive devices, with failure rates of $<\mathbf{0 . 8 \%}$ and almost no user action, have extremely poor uptake. Recognizing that the variation of side effect profiles will also impact use, this disconnect between efficacy, effort and use suggests that forces beyond the intrinsic merits of a contraceptive system play a role in these decisions. Cost and accessibility can both inhibit the use of intrauterine systems, which are a large upfront investment and require physician insertion. Peers, the media and providers all influence contraceptive decision-making and may also contribute their own bias. We advocate for providing public funding for all contraceptive options as well as engaging in open discussion with both patients and the public to improve our delivery of reproductive health care.

\section{INTRODUCTION}

Contraceptive choice is complex and must meet a woman's needs while ensuring efficacy, reliability and safety. The woman must possess (1) knowledge on method choices, (2) motivation to pursue contraception and (3) the ability to access her preferred method(s). These factors then interact with external influences such as affordability, access to healthcare, and a woman's social context. Further, contraceptive needs may change over a woman's reproductive life span based on personal and family dynamics. ${ }^{1}$

Despite the existence of highly effective contraception, $41 \%$ of 208 million pregnancies worldwide were unintended in 2008, with the highest rates occurring in Eastern and Middle Africa. ${ }^{2}$ In Canada there are greater than 180000 unplanned pregnancies per year with an estimated health-care cost of $\$ 320$ million. ${ }^{3}$ Unintended pregnancies can be associated with negative mental, physical, social and economic outcomes for women and their families. These include increased risk of perinatal depression and partner violence, decreased likelihood of reaching education or career goals, and economic instability. ${ }^{4-6}$ In addition, women having unplanned pregnancies are more likely to suffer from inadequate prenatal care and nutrition, increased high-risk behavior such as exposure to tobacco, alcohol or illicit drugs, low-birth weight infants, and increased infant mortality. ${ }^{78}$ When women are able to plan and space their pregnancies they participate more actively in the economy while experiencing improved health outcomes for themselves and their families. ${ }^{9}$

\section{CONTRACEPTIVE OPTIONS AND USE}

There are many reversible contraceptive options, which can provide a woman with the freedom to decide if and when she will become pregnant without permanently altering her reproductive potential. Reversible contraceptives are categorized into barrier methods, short-acting reversible contraceptives (SARCs), and long-acting reversible contraceptives (LARCs). Barrier methods, including the male and female condom, prevent sperm entry into the female upper genital tract. While the importance of condoms for the prevention of sexually transmitted infections (STIs) cannot be overstated, their failure rate for pregnancy prevention is $2 \%$ for male and $5 \%$ for female condoms with perfect use. These rates rise to $18 \%$ and $21 \%$ respectively with typical use (Table 1). ${ }^{10,11}$ SARCs are progestin preparations with or without estrogen, which prevent ovulation. Examples include the daily combined oral contraceptive pill (OCP), weekly applied contraceptive patch, monthly vaginal ring, and quarterly contraceptive injections. ${ }^{11}$ These methods provide increased protection against pregnancy when compared to barrier methods but remain vulnerable to errors in execution. Estrogen-containing SARCs may also increase a woman's thromboembolic risk. ${ }^{11}$ With perfect use, SARC failure rates are $0.2 \%$ to $0.3 \%$. With typical use, those failure rates rise to $9 \%$ for the OCP, patch, and ring, and $6 \%$ for injections (Table 1$).{ }^{10}$ Narrowing the gap between perfect and typical use failure rates with quarterly injections suggests that decreasing the level of user action increases a method's real-world efficacy. This is further supported when considering LARCs where user action is almost entirely absent. LARCs are available in two forms in Canada, the copper intrauterine device (IUD) and the levonorgestrel intrauterine system (LNG-IUS). ${ }^{11}$ Both systems alter the uterine environment to prevent conception and implantation. ${ }^{2}$ With these alterations there are menstrual irregularities; amenorrhea is common for the LNG-IUS, while increased flow is often experienced with copper containing IUDs. These devices must be inserted into the uterus, which carries some small risks including perforation. Once inserted LARCs can provide pregnancy protection for up to 10 years, depending on the device. ${ }^{11,12}$ The IUD has perfect and typical use failure rates of $0.6 \%$ and $0.8 \%$ respectively while the LNG-IUS has equal perfect and typical use failure rates of $0.2 \%$, which are comparable to tubal liga- 
tion (Table 1) ${ }^{10}$ Considering the clear contraceptive advantages of LARCs their uptake in Canada is surprisingly low. ${ }^{13}$

In Canada, $73 \%$ of married or in union women use contraception. While many North American couples choose permanent sterilization, by far the most common reversible methods are the male condom and OCP with estimated prevalence rates of $15 \%$ and $21 \%$ respectively. These methods are similarly prevalent in the United States and Europe (Figure 1). However, the use of LARCs in Canada lags behind, representing only $1 \%$ of use compared to $5 \%$ in the United States and $11 \%$ in Europe. It is worth noting that Canada's $1 \%$ prevalence is on par with that of the WHO's 48 least developed countries. $^{13}$

Table 1. Contraceptive failure rates during perfect and typical use and frequency of action. ${ }^{10-12}$

\begin{tabular}{|c|c|c|c|}
\hline \multirow[b]{2}{*}{ Method } & \multicolumn{2}{|c|}{ Failure (\%) } & \multirow[b]{2}{*}{ Required frequency of action } \\
\hline & Perfect Use & Typical Use & \\
\hline OCP & 0.3 & 9.0 & Daily \\
\hline Patch & & & Weekly \\
\hline Vaginal ring & & & Monthly \\
\hline Contraceptive injections & 0.2 & 6.0 & Quarterly \\
\hline IUD & 0.6 & 0.8 & Up to 10 years \\
\hline LNG-IUS & 0.2 & 0.2 & Up to 5 years \\
\hline Male condom & 2.0 & 18.0 & Every encounter for \\
\hline Female condom & 5.0 & 21.0 & STI protection \\
\hline
\end{tabular}

\section{COMPLEXITIES IN CONTRACEPTIVE CHOICE}

Contraceptive decision-making should be a personal choice, rooted in a woman's fundamental right to self-determination. However, there are external forces such as cost, availability, culture and social situation that may have undue influence. Cost is often limiting, particularly when considering the upfront expense of the LNGIUS at over $\$ 300$. This is substantially greater than OCPs at $\sim \$ 11$ per month but less than the combined cost of OCPs over the 5-year lifespan of some LNG-IUSs. ${ }^{3}$ Individual users or private insurers in Canada must often absorb these costs. As of 2009 Quebec was the only province that reimbursed all hormonal contraceptive methods through a provincial plan. This may contribute to the higher prevalence of LNG-IUSs in Quebec relative to other provinces. ${ }^{14}$ Mathematical modeling of the Canadian and American healthcare systems showed that a proportional increase in LARC use over OCP is cost saving after 1 to 1.5 years through the elimination of unplanned pregnancy due to poor adherence., 3,15 These models highlight potential systemic gains of publicly funding the full range of contraceptive options. ${ }^{3}$

These decisions are also impacted by local availability of services. Condom and OCP use in Canada far exceed other reversible methods. While this may stem from their wide availability and familiarity, Black et al. in their 2009 national survey suggest that accessibility contributes significantly to the dramatically lower prevalence of other systems, particularly LARCs. Trained healthcare providers are needed for insertion of LARCs, which are often not available in rural and remote regions of Canada. This is of particular

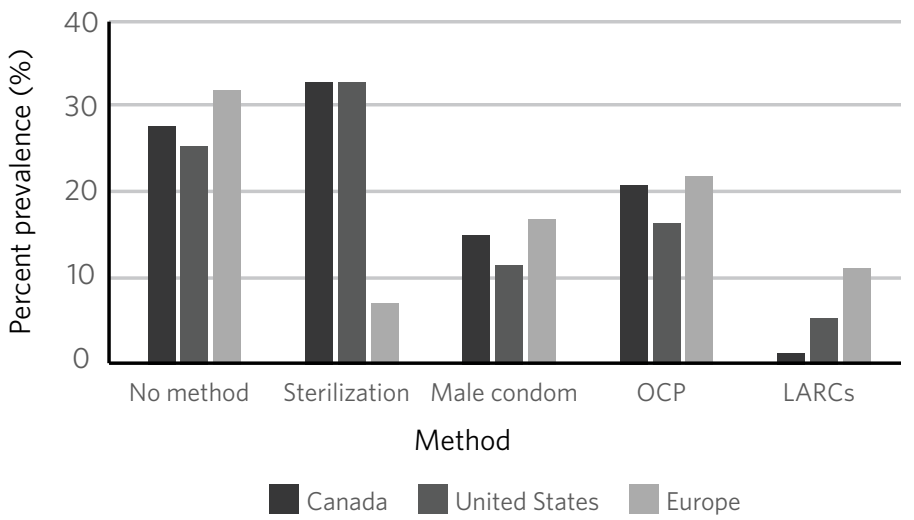

Figure 1. Estimated prevalence of contraceptive methods in among married or in union women in Canada (black), the United States (grey), and Europe (white) in 2015. ${ }^{13}$

note when considering First Nations populations who are disproportionately affected by this disparity in reproductive choice. ${ }^{14}$

Outside of the logistics of obtaining a contraceptive method are cultural and societal influences that shape what are considered acceptable forms of contraception. Cultural and religious values have enormous impacts on family planning goals, stressing the importance of taking a broad and unbiased approach to contraceptive counselling. ${ }^{16}$ An individual is also likely to depend on their social network to guide their contraceptive choices. In a Nigerian study, $98 \%$ of males and $85 \%$ females in a sample of 313 unmarried students based their contraceptive choices on peer information. ${ }^{17}$ In this study peer-to-peer spread of information led to use of less reliable contraceptive options, underlining both the power of peer influence and importance of accurate health messaging.

Health messaging in the media has the capacity to reach a wide audience but also to propagate misinformation. When side effect profiles are involved this misinformation can damage the public perception of a method and may result in non-use. ${ }^{3,18}$ This is illustrated by the case of the Dalkon Shield, a plastic intrauterine device that was approved by the FDA in 1968 and used by 3.2 million women in the United States and abroad. It was removed from the market in 1974 when it was shown to increase rates of pelvic inflammatory disease and related complications leading to at least 10 deaths and over 200000 legal claims. ${ }^{19,20}$ This led to intrauterine contraception largely disappearing from the US market, despite low risks with current devices. ${ }^{12}$ In addition to lasting concerns associated with the safety of intrauterine devices, other unsubstantiated claims have negatively impacted LARC use in North America. A survey in California demonstrated providers often did not recommend LARCs to groups of women, including those who were nulliparous or post abortion, due to unsupported beliefs that they were unsuitable candidates for the systems. ${ }^{21}$ This highlights how health providers can also be unduly influenced by popular opinion.

\section{CONCLUSION}

It is a woman's right to protect herself against unplanned pregnancy. ${ }^{22-24}$ However, there is no one contraceptive method that is 
right for everyone. Ultimately, we should aim to facilitate a system where a woman is free to make the informed choice that best suits her contraceptive needs with the side effect profile that is most acceptable to her. Reliable contraception should be within reach for every Canadian woman but cost, accessibility and availability of information continue to inhibit progress. We advocate for public subsidization of all contraceptive methods in addition to more active discussions with the public and patients surrounding contraceptive choice. In these ways we strive to more effectively support women as they decide under what circumstances they choose to become pregnant.

\section{REFERENCES}

1. Black A, Guilbert E. Canadian Contraception Consensus. SOGC Clin Pract Guidel. 2015;Oct(329):1-28.

2. Singh S, Sedgh G, Hussain R. Unintended Pregnancy: Worldwide Levels, Trends, and Outcomes. Stud Fam Plann. 2010;41(4):241-50.

3. Black AY, Guilbert E, Hassan F, et al. The Cost of Unintended Pregnancies in Canada : Estimating Direct Cost, Role of Imperfect Adherence, and the Potential Impact of Increased Use of Long-Acting Reversible Contraceptives. J Obstet Gynaecol Canada. 2015;37(12):1086-87.

4. Abajobir AA, Maravilla JC, Alati R, et al. A systematic review and meta-analysis of the association between unintended pregnancy and perinatal depression. J Affect Disord. 2016;192:56-63.

5. Martin-de-las-Heras S, Velasco C, Luna J de D, et al. Unintended pregnancy and intimate partner violence around pregnancy in a population-based study. Women Birth. 2015;28(2):101-5.

6. Institute of Medicine (US) Committee on Unintended Pregnancy. The Best Intentions: Unintended Pregnancy and the Well-Being of Children and Families. (Brown S, Eisenberg L, eds.). Washington, DC: National Academies Press (US); 1995.

7. Cheng D, Schwarz EB, Douglas E, et al. Unintended pregnancy and associated maternal preconception, prenatal and postpartum behaviors. Contraception. 2009;79(3):194-8.

8. Terplan M, Cheng D, Chisolm MS. The relationship between pregnancy intention and alcohol use behavior: An analysis of PRAMS data. J Subst Abuse Treat. 2014;46(4):506-10.

9. Richards C. Protecting and Expanding Access to Birth Control. N Engl J Med. 2016;374(9):801-3.

10. Trussell J. Contraceptive failure in the United States. Contraception. 2011;83(5):397-404.

11. Fisher WA, Black A. Contraception in Canada: A review of method choices, characteristics, adherence and approaches to counselling. CMAJ. 2007;176(7):953-61.

12. Stoddard A, McNicholas C, Peipert JF. Efficacy and safety of long-acting reversible contraception. Drugs. 2011;71(8):969-80.

13. United Nations Department of Economic and Social Affairs, Population Division (2015) Trends in Contraceptive Use Worldwide 2015 (ST/ESA/SER.A/349)

14. Black A, Yang Q, Wu Wen S, et al. Contraceptive use among Canadian women of reproductive age: results of a national survey. J Obstet Gynaecol Canada. 2009;31(7):627-40.

15. Trussell J, Henry N, Hassan F, et al. Burden of unintended pregnancy in the United States: Potential savings with increased use of long-Acting reversible contraception. Contraception. 2013;87(2):154-61.

16. Srikanthan A, Reid RL. Religious and cultural influences on contraception. J Obstet Gynaecol (Lahore). 2008;30(2):129-37.
17. Iyoke CA, Ezugwu FO, Lawani OL, et al. Peer-driven contraceptive choices and preferences for contraceptive methods among students of tertiary educational institutions in Enugu, Nigeria. Patient Prefer Adherence. 2014;8:1043-50.

18. Sedgh G, Hussain R. Reasons for Contraceptive Nonuse among Women Having Unmet Need for Contraception in Developing Countries. Stud Fam Plann. 2014;45(2):151-69.

19. Pendergast P, Hirsh HL. The Dalkon Shield in perspective. Med Law. 1986;5(1):35-44.

20. Byrne K. Medical records in litigation: the Dalkon Shield story. AMRO. 1992;32(5):11-4.

21. Harper CC, Blum M, de Bocanegra HT, et al. Challenges in translating evidence to practice: the provision of intrauterine contraception. Obstet Gynecol. 2008;111(6):1359-69.

22. United Nations. International Conference on Population Development: Program of Action, 20th Anniversary Edition [Internet]. New York, NY: UNFPA; 2014 [cited 2016 May 8]. Available from: http://www. unfpa.org/publications/international-conference-population-and-development-programme-action

23. United Nations and Centre for Reproductive Rights. The right to contraceptive information and services for women and adolescents: Briefing paper [Internet]. New York, NY: United Nations and Centre for Reproductive Rights; 2014 [cited 2016 May 29]. Available from: http:// www.unfpa.org/resources/rights-contraceptive-information-and-services-women-and-adolescents

24. United Nations. Convention on the Elimination of All Forms of Discrimination against Women, art. 16, para. 1 [Internet]. New York, NY: United Nations; 1979 Dec 18 [cited 2016 May 29]. Available from: http://www.un.org/womenwatch/daw/cedaw/text/econvention.htm 